\title{
Noncharacteristic half-lives in radioactive decay
}

\author{
Álvaro Corral, ${ }^{1}$ Francesc Font, ${ }^{1}$ and Juan Camacho ${ }^{2}$ \\ ${ }^{1}$ Centre de Recerca Matemàtica, Edifici Cc, Campus Bellaterra, E-08193 Barcelona, Spain \\ ${ }^{2}$ Grup FESTA, Facultat de Ciències, Universitat Autònoma de Barcelona, E-08193 Barcelona, Spain
}

(Received 27 December 2010; revised manuscript received 24 April 2011; published 6 June 2011)

\begin{abstract}
Half-lives of radionuclides span more than 50 orders of magnitude. We characterize the probability distribution of this broad-range data set at the same time that we explore a method for fitting power laws and testing goodness-of-fit. It is found that the procedure proposed recently by Clauset et al. [SIAM Rev. 51, 661 (2009)] does not perform well as it rejects the power-law hypothesis even for power-law synthetic data. In contrast, we establish the existence of a power-law exponent with a value around 1.1 for the half-life density, which can be explained by the sharp relationship between decay rate and released energy, for different disintegration types. For the case of alpha emission, this relationship constitutes an original mechanism of power-law generation.
\end{abstract}

DOI: 10.1103/PhysRevE.83.066103

PACS number(s): 89.75.Da, 23.90.+w

\section{INTRODUCTION}

It is well known that most processes in physics are governed by a characteristic scale. Radioactive decay is a prototypical example of this; for a given nuclide (i.e., an isotope of an element in a given energy state), any nucleus has the same constant probability of disintegration per unit time, which allows one to define the half-life $t_{1 / 2}$ of the nuclide (or, equivalently, its lifetime), setting the time scale of the decay [1].

It is noteworthy that these half-lives take very disparate values, from very small fractions of a second to many millions of years (for example, $164 \mu \mathrm{s}$ for ${ }^{214} \mathrm{Po}$ and $4.47 \times 10^{9}$ years for ${ }^{238} \mathrm{U}$ ). One can wonder if there is some predominant scale for the half-lives of the radionuclides, or, on the contrary, if half-lives can be considered as scale free, and what could be the physical reasons for that behavior. It is remarkable that in the past decades, many phenomena that violate the usual requirement of the existence of a characteristic scale have been found in physics and beyond [2-6], under the form of power-law functions, the hallmark of scale invariance. In parallel, the enormous range of values covered by the nuclear half-lives will allow us to test the performance of a recently proposed statistical tool to fit power-law distributions and to provide the goodness of such fits [7].

\section{DATA}

We analyze data coming from the Lund/LBNL Nuclear Data Search web page [8]. A total of 3032 nuclides are found from a query in the range $10^{-34} \mathrm{~s}<t_{1 / 2}<10^{34} \mathrm{~s}$ (to avoid 249 "stable" nuclides as well as 433 ones with unknown half-lives, which are treated as having zero half-life in the web engine). Thirty of these nuclides do not have a well-defined half-life, as $t_{1 / 2}$ is bounded only from above or from below. These, as well as the neutron (which is not a radionuclide), are excluded from the analysis. On the other hand, we include by hand the isotope ${ }^{209} \mathrm{Bi}$, which was previously thought to be the heaviest stable nuclide but has recently been found to be unstable, with $t_{1 / 2}=1.9 \times 10^{19}$ years for $\alpha$ emission [9]. We deal then with 3002 unstable nuclides, 723 of which are metastable isomers (excited states with a "relatively" long half-life) and the rest, 2279 , corresponding to ground states. As we have found no big difference between the statistics of the ground states and the isomers, we have considered both types of radionuclides together in most of the analysis. For a few of them (17) the halflife comes in units of energy, $E$, which can be converted into half-life using the formula $t_{1 / 2} E=\hbar$ (where $\hbar$ is the reduced Planck's constant). This yields values of $t_{1 / 2}$ below $10^{-16} \mathrm{~s}$.

\section{DATA ANALYSIS}

A simple plot of the half-life probability density on a log-log scale, displayed in Fig. 1, shows that the most prominent signature of the distribution is a linear trend for a certain range of half-lives, an indication of a possible power-law behavior. So, our first step is to test the existence of a power-law distribution above a certain cutoff value $a$ of the half-life, i.e.,

$$
D(t) \propto \frac{1}{t^{\tau}} \quad \text { for } t \geqslant a,
$$

where $\propto$ denotes proportionality, $D(t)$ is the probability density of the half-life, from now on denoted as $t$, and the exponent verifies $\tau>1$ (for normalization).

We try the method proposed by Clauset et al. [7], which looks for the value of $a$ which minimizes the KolmogorovSmirnov (KS) distance [11] between the empirical cumulative distribution and the theoretical fit obtained by maximumlikelihood estimation of $\tau$ (values of $t$ below $a$ do not play any role in the fit of $\tau$ but are important for the estimation of $a$ ). Once the values of $\tau$ and $a$ are found, a $p$ value is calculated, giving the probability that true power-law distributed data, with the same exponent and cutoff as the ones estimated for the empirical data, have a KS distance with its fit larger than the distance of the empirical data with its fit. This is done by means of Monte Carlo simulations of the resulting distribution, applying to the synthetic data exactly the same fitting procedure as the one used for the empirical data (maximum-likelihood estimation and minimization of the KS distance), in order to avoid biases in the $p$ value $[7,12]$.

The half-life distribution is easily simulated in the powerlaw range (by means for instance of the inversion method [11]), but it is necessary also to simulate it outside this range in order to optimize the value of $a$ in the synthetic data (recall that the simulated distribution has to be treated in the same way as 


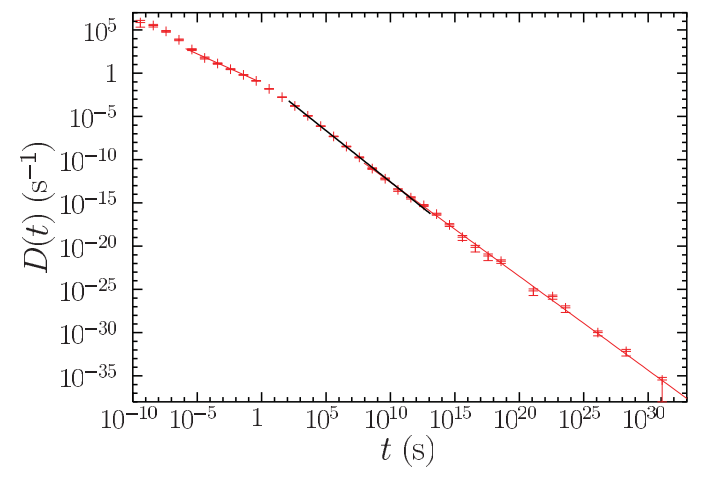

FIG. 1. (Color online) Empirical probability density of the halflives $(t)$ of the 3002 radionuclides available (19 values below $10^{-10} \mathrm{~s}$ are not shown). The straight lines are three of the power-law fits explained in the text, covering the fitting range obtained by the minimization of the KS distance. The exponents are $\tau=0.67,1.19$, and 1.09 (from left to right). Note that extreme caution should be present when estimating a probability density using large bins; see Ref. [10].

the empirical one). This is simply done by a kind of bootstrap method [7], where the synthetic values of $t$ are taken randomly, with replacement, from the empirical data in that range (i.e., $t<a$ ). Details of the fitting and the goodness-of-fit testing are explained in an appendix. Noticeably, the whole fitting and testing procedure does not use that $D(t)$ should be a straight line in a log-log scale.

The results of this method applied to the nuclear half-lives yield the values $a=29.85 \mathrm{~s}$ and $\tau=1.16$ with a KS distance $d_{m}=0.036$ but with $p=0$ (from 1000 simulations, that is, in no case a KS distance larger than the empirical 0.036 was found). So, the direct application of the Clauset et al.'s method leads to the rejection of the power-law hypothesis. However, Fig. 2(a) shows that this result is not convincing. We plot there, as a function of the cutoff $a$, the KS distance and the $p$ value corresponding to the case in which $a$ were fixed or known (we denote the $p$ value for this case as $q$, in order to distinguish it from the $p$ value when $a$ is optimized). It is clear that the power-law fit must be rejected for any value of $a$ below $10^{7} \mathrm{~s}$ (as $\left.q=0\right)$, but above this value $q$ takes nonzero values, fluctuating between zero and 1 , as it would correspond to true power-law distributed data.

So, although the Clauset et al.'s method fails when applied to the whole data set, we try to apply it now to a restricted data set, considering a range of variation of the parameter $a$ above $1000 \mathrm{~s}$ (to avoid the misleading minimum of the $\mathrm{KS}$ distance at around $30 \mathrm{~s}$ ). This leads to a new minimum at $a=8.8 \times 10^{7} \mathrm{~s}$ (close to 3 years) and an exponent $\tau=$ $1.09 \pm 0.01$ with $d_{m}=0.052$ and $p=33 \% \pm 5 \%$. This is an acceptable result, which means in fact that we cannot reject the power-law hypothesis. Given that the maximum half-life is larger than $10^{31} \mathrm{~s}$ (for ${ }^{128} \mathrm{Te}$ ), this power law spans more than 23 orders of magnitude. However, one can realize that there are only 128 nuclides in the power-law range, which makes its relevance as a characterization of nuclear properties rather limited. In any case, we have found a power-law tail for the distribution of nuclear half-lives, together with the conclusion that the blind application of Clauset et al.'s method is not reliable. The condition $a>1000 \mathrm{~s}$ is not determinant, as other values lead to very similar results; see Table I.

In order to confirm the failure of Clauset et al.'s method we apply the absolute minimization of the KS distance to simulated data, with a power-law distribution with $\tau=1.09$ above $a=8.8 \times 10^{7} \mathrm{~s}$ and bootstrapping the empirical data below that value [7]; the $\mathrm{KS}$ distance corresponding to two of them is shown in Fig. 2(b). In $80 \%$ of the simulations the $p$ value turns out to be zero, leading to the rejection of the powerlaw hypothesis when the data have, by construction, a true power-law range. Note that in this case, as the null hypothesis is true, the $p$ value should be uniformly distributed between 0 and 1. Therefore, we have come across a counterexample that invalidates the general applicability of the Clauset et al.'s method. There is nothing surprising here, as, after all, these
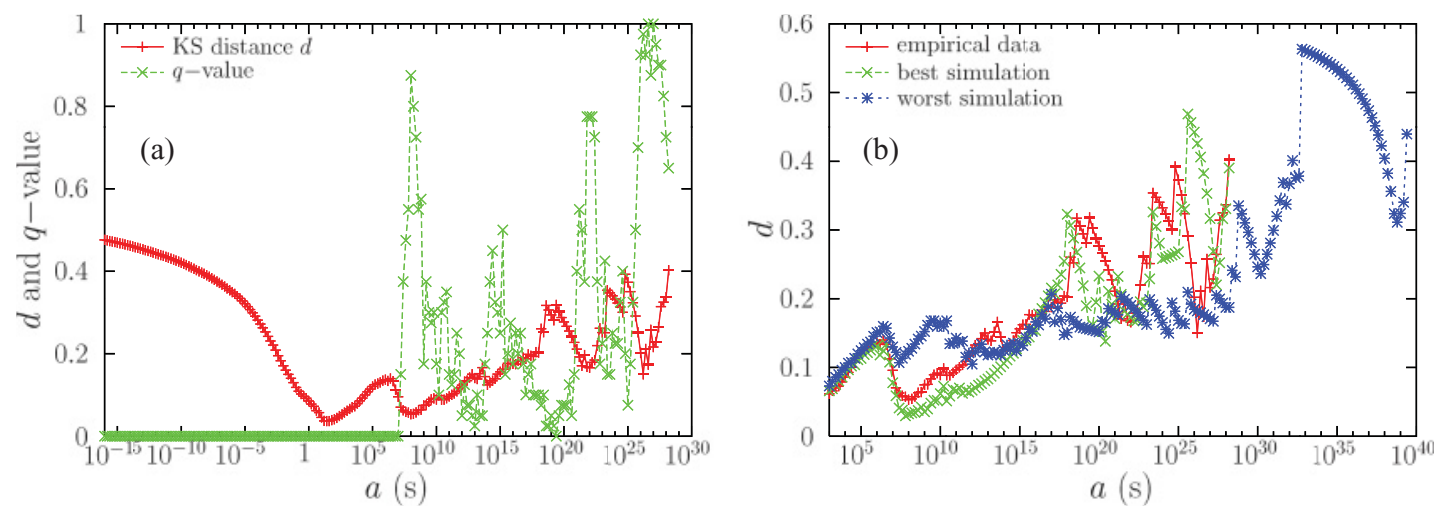

FIG. 2. (Color online) (a) Kolmogorov-Smirnov distance $d$ for different values of the lower cutoff $a$ in a power-law fit of the radionuclide half-life distribution, together with the associated " $q$ value", which would be identical to the $p$ value if the parameter $a$ were not optimized. As $a$ is also variable in the fit, the $q$ value overestimates the true $p$ value. It is clear that the absolute minimum of $d$ yields $q=0$ and therefore $p=0$, but for $a>10^{7} \mathrm{~s}$ the $q$ value becomes nonzero. (b) Comparison of the empirical KS distance in (a) with that from simulations of a power law with exponent $\tau=1.09$ for $t>8.8 \times 10^{7} \mathrm{~s}$ (and with random values taken from the empirical distribution below that value, as explained in the text). Only the simulations with the smallest $\left(d_{m}=0.028\right.$, best) and largest KS distances $(0.073$, worst $)$ out of 100 simulations are shown. The fraction of minimum distances above the empirical value 0.052 defines the $p$ value. 
TABLE I. Results of several power-law fits and goodness of fit for the half-lives of the 3002 radionuclides available. The results for the non-upper-truncated power law $(b=\infty)$ are calculated with a resolution of 100 points per decade in $a$, whereas for the upper-truncated power law we use 5 points per decade in $a$ and $b . N_{a b}$ is the number of nuclides in the range $a \leqslant t<b, c$ is the value of the proportionality constant of the power law needed to fit the whole distribution in Fig. 1 (when $t$ is measured in seconds), and $\varepsilon$ is one standard deviation in the distribution of the maximum-likelihood estimation of the exponent $\tau$, see Ref. [29]. The $p$ value is obtained from 100 simulations, and its uncertainty is calculated as in Ref. [14]. The value $p=0$ in the first row is maintained in 1000 simulations.

\begin{tabular}{|c|c|c|c|c|c|c|c|c|}
\hline Condition & $a(\mathrm{~s})$ & $b(\mathrm{~s})$ & $b / a$ & $N_{a b}$ & $c$ & $\tau \pm \varepsilon$ & $d_{m}$ & $p(\%)$ \\
\hline$b=\infty$ & 29.85 & $\infty$ & $\infty$ & 1624 & 0.145 & $1.16 \pm 0.01$ & 0.036 & 0 \\
\hline$b=\infty, a>10^{3} \mathrm{~s}$ & $8.8 \times 10^{7}$ & $\infty$ & $\infty$ & 128 & 0.018 & $1.09 \pm 0.01$ & 0.052 & $33 \pm 5$ \\
\hline$b=\infty, a>10^{4} \mathrm{~s}$ & $8.8 \times 10^{7}$ & $\infty$ & $\infty$ & 128 & 0.018 & $1.09 \pm 0.01$ & 0.052 & $37 \pm 5$ \\
\hline$b=\infty, a>10^{5} \mathrm{~s}$ & $8.8 \times 10^{7}$ & $\infty$ & $\infty$ & 128 & 0.018 & $1.09 \pm 0.01$ & 0.052 & $37 \pm 5$ \\
\hline$a>1 \mathrm{~s}$ & 126 & $7.9 \times 10^{6}$ & $6.3 \times 10^{4}$ & 1138 & 0.185 & $1.18 \pm 0.01$ & 0.012 & $88 \pm 3$ \\
\hline$b / a>10^{8}$ & 126 & $1.3 \times 10^{13}$ & $1.0 \times 10^{11}$ & 1277 & 0.198 & $1.19 \pm 0.01$ & 0.015 & $39 \pm 5$ \\
\hline$b<1 \mathrm{~s}$ & $5.0 \times 10^{-5}$ & 0.32 & $6.3 \times 10^{3}$ & 460 & 0.085 & $0.65 \pm 0.02$ & 0.023 & $40 \pm 5$ \\
\hline$b<1 \mathrm{~s}, b / a>10^{4}$ & $1.3 \times 10^{-6}$ & 0.50 & $4.0 \times 10^{5}$ & 559 & 0.079 & $0.67 \pm 0.02$ & 0.024 & $32 \pm 5$ \\
\hline
\end{tabular}

authors do not provide any theoretical justification about why their ad-hoc method should work, and only check its performance (under controlled conditions) in simple synthetic examples.

Our second option for the distribution of half-lives is the use of an upper truncated power-law distribution,

$$
D(t) \propto \frac{1}{t^{\tau}} \quad \text { for } a \leqslant t<b,
$$

where $a$ and $b$ are the lower and upper cutoffs, respectively, with no condition on the value of $\tau$ this time. Some examples of the applicability of this distribution can be found in Ref. [13]. In this case we need to generalize Clauset et al.'s method (a detailed description is provided in the supplementary information of Ref. [14], of which our appendix gives a summary). The basic idea is again to find the values of $a$ and $b$ which minimize the KS distance between the empirical distribution and its fit. An important difference with the previous case is that when $b$ is not infinite, several power-law pieces can coexist in the data, and a criterion is necessary to select one of them. Restricting this time the $a$ value to $a>1 \mathrm{~s}$, the pair that minimizes the KS distance (determined with a resolution of 5 points per decade) is $a=126 \mathrm{~s}$ and $b=7.9 \times 10^{6} \mathrm{~s}$, with $\tau=1.18 \pm 0.01, d_{m}=0.012$, and $p=88 \% \pm 3 \%$. Therefore, a power law ranging more than 4 decades cannot be rejected.

However, this solution, with its high $p$ value, is not fully satisfactory, as its range is a small fraction of the total half-life range. We have observed, by means of computer simulations, that the generalization of the Clauset et al.'s method that we are using leads to severe underestimations of the value of $b$, of many orders of magnitude in some realizations. Adding a different restriction, for example, $b / a>10^{8}$, we find the conditional minimum at $a=126 \mathrm{~s}$ and $b=1.3 \times 10^{13} \mathrm{~s}$, with an exponent $\tau=1.19 \pm 0.01$, a KS distance $d_{m}=0.015$ and a $p$ value $p=39 \% \pm 5 \%$, comprising 1277 different nuclides. We conclude that a power law over 11 orders of magnitude is an acceptable fit (although higher values of $b$ still yield not too low $p$ values). All the fits are collected in Table I and the most representative ones are shown in Fig. 1 (see caption for details). A more standard analysis based on the calculation of the $q$ values (again, $p$ values where $a$ and $b$ are fixed, as in Ref. [15]) show that indeed these power-law fits are acceptable.

The results found so far can be summarized by two power-law ranges, one from, roughly, $t=100$ to $10^{10} \mathrm{~s}$, with $\tau=1.19$, and another one for $t$ above $10^{10} \mathrm{~s}$, with $\tau=1.09$ (the crossover region between the two exponents is difficult to discriminate). The close value of the exponents lead us to speculate that the two regimes could be in fact the same, with some artifact causing the small but significant difference between their values. After all, we have studied about 3000 nuclides, but of course these do not constitute a complete sample. Among the nuclides considered as stable, more than 100 are theoretically unstable, but with a half-life that has not been possible to measure. And, of course, not all nuclides that may exist have been synthesized so far [16]. So, we leave the door open to the fact that a unique exponent around $\tau=1.15 \pm 0.10$ could describe the complete set of radioactive half-lives above the range of a few minutes. This would imply the existence of scale invariance for about 30 orders of magnitude.

It is interesting to see how these results are affected by the mode of disintegration of the radionuclides. Different decays involve different processes and even different types of interaction (strong for $\alpha$ emission and weak for $\beta$, for instance). We can restrict the half-life statistics to nuclides that decay in a particular way. Table II shows the results corresponding to the $\alpha, \beta$, electron-capture, and isomeric-transition decays, including also the separation of all nuclides into isomers and ground states. To be unambiguous, when we consider $\alpha$ disintegration, only those radionuclides that decay exclusively by $\alpha$ emission are considered, and the same for the other types of disintegration. We fit a double-truncated power-law distribution concentrating on the region of large half-lives $(a>1 \mathrm{~s})$ and see that in all cases the tail of the distribution is compatible with a power law with an exponent between 1 and 1.3 .

\section{ORIGIN OF POWER-LAW BEHAVIOR}

In the case of $\alpha$ decay we can give a simple explanation of the exponent. Gamow's model assumes the pre-existence of an $\alpha$ particle inside a potential well [1]. Quantum tunneling of the 
TABLE II. Results of a power-law fit to the half-lives of nuclides in their ground or excited states (isomers) and to the nuclides that follow a certain unique decay type. The fits are conditioned to $a>1 \mathrm{~s}$ and $b / a>10^{8}$. EC denotes orbital electron capture and IT, isomeric transition ( $\gamma$ ray or conversion electron emission from an exited state). Other disintegration types yield very low numbers of nuclides, $N$. Rest of symbols are as in the previous table.

\begin{tabular}{|c|c|c|c|c|c|c|c|c|c|}
\hline Case & $N$ & $a(\mathrm{~s})$ & $b(\mathrm{~s})$ & $b / a$ & $N_{a b}$ & $\mathrm{c}$ & $\tau \pm \varepsilon$ & $d_{m}$ & $p(\%)$ \\
\hline All & 3002 & 126 & $1.3 \times 10^{13}$ & $1.0 \times 10^{11}$ & 1277 & 0.198 & $1.19 \pm 0.01$ & 0.015 & $39 \pm 5$ \\
\hline Ground & 2279 & 126 & $3.2 \times 10^{13}$ & $2.5 \times 10^{11}$ & 1009 & 0.182 & $1.175 \pm 0.01$ & 0.020 & $18 \pm 4$ \\
\hline isomers & 723 & 200 & $7.9 \times 10^{11}$ & $4.0 \times 10^{9}$ & 248 & 0.338 & $1.25 \pm 0.02$ & 0.038 & $25 \pm 4$ \\
\hline$\alpha$ & 170 & 126 & $3.2 \times 10^{25}$ & $2.5 \times 10^{23}$ & 22 & 0.003 & $1.00 \pm 0.01$ & 0.082 & $69 \pm 5$ \\
\hline$\beta^{+}$ & 607 & 501 & $3.2 \times 10^{13}$ & $6.3 \times 10^{10}$ & 281 & 0.833 & $1.29 \pm 0.02$ & 0.036 & $30 \pm 5$ \\
\hline$\beta^{-}$ & 688 & 126 & $3.2 \times 10^{17}$ & $2.5 \times 10^{15}$ & 369 & 0.251 & $1.19 \pm 0.01$ & 0.028 & $37 \pm 5$ \\
\hline $\mathrm{EC}$ & 82 & $2 \times 10^{5}$ & $5.0 \times 10^{13}$ & $2.5 \times 10^{8}$ & 62 & 2.289 & $1.22 \pm 0.03$ & 0.089 & $6 \pm 2$ \\
\hline IT & 192 & 2 & $7.9 \times 10^{9}$ & $4.0 \times 10^{9}$ & 106 & 0.103 & $1.16 \pm 0.02$ & 0.053 & $42 \pm 5$ \\
\hline
\end{tabular}

particle leads to a very sharp relation between the half-life and the energy $Q$ released in the emission (which can be related to the Geiger-Nuttall rule),

$$
t=A \exp \left(\frac{B Z}{\sqrt{Q}}\right)
$$

where $Z$ is the atomic number of the resulting nucleus, $B$ is a positive constant, and $A$ can be considered as a constant, too, in a first approximation [17]. Defining $U=Q / Z^{2}$, with an unknown probability density $f(U)$, conservation of probability leads to

$$
D_{\alpha}(t)=f(U)\left|\frac{d U}{d t}\right|=f\left(\frac{B^{2}}{\ln ^{2} t / A}\right)\left(\frac{2 B^{2}}{\ln ^{3} t / A}\right) \frac{1}{t},
$$

where the factor $F(t)$ that multiplies the hyperbolic $1 / t$ tail constitutes a slowly varying function for a broad class of choices for $f(U)$ [i.e., $F(\ell t) / F(t) \rightarrow 1, \forall \ell>0$ when $t \rightarrow$ $\infty$ ]. For example, $f(U)$ could be uniform, linear, parabolic, hyperbolic, etc., with no influence on the $1 / t$ asymptotic tail. This result is in surprising good agreement with the empirical exponent found for the $\alpha$ decay, $\tau=1.0$; see Table II. Note that the present mechanism for power-law generation has some resemblance with the ones collected in Refs. [6,18], but, in contrast to them, it is not derived neither from another power-law relationship between $t$ and $U$ (see next) nor from an exponential energy-barrier distribution.

For the $\beta$ decay a similar argument is possible. In this case, Sargent rule establishes a different relationship between $t$ and the released energy $Q$ [19], which, in a very crude approximation, could be considered as a proportionality between $t$ and $1 / Q^{5}$, so

$$
Q \simeq \frac{C}{t^{1 / 5}}
$$

Then, the distribution of $t$ could be obtained from that of $Q$, $g(Q)$,

$$
D_{\beta}(t) \propto g\left(\frac{C}{t^{1 / 5}}\right) \frac{1}{t^{1+1 / 5}},
$$

which yields asymptotically a power law with exponent 1.2 if $g$ is a slowly varying function of $t$.

However, the fact that $C$ could be constant is far from true; rather, it varies in a range of many orders of magnitude for different nuclides [1]. A mathematical theorem guarantees that, under very general conditions, the values above a high threshold of a random variable tend to follow the so-called generalized Pareto distribution [20,21], which asymptotically yields a simple power-law tail when the range of variation is broad (decay slower than an exponential). So, it is reasonable to assume that, over some threshold value, the half-life is given by the product of two power-law variables, one with an exponent $6 / 5$ (derived above from Sargent rule) and the other one unknown, $1+v$. Considering independence between both factors, the distribution of the product is given by the convolution of the logarithms of each factor, which turn out to be exponentially distributed. The result is,

$$
D_{\beta}(t) \simeq \frac{v / 5}{v-1 / 5}\left(\frac{K^{1 / 5}}{t^{6 / 5}}-\frac{K^{v}}{t^{1+v}}\right),
$$

where $K$ is the minimum value of $t$ for which this behavior holds. This yields a power-law tail with exponent $\tau=1+1 / 5$ if $v>1 / 5$ and with exponent $\tau=1+v$ if $v<1 / 5$ (but for normalization, $v>0$ ). In any case, the exponent $\tau$ of the tail is constrained in the range $(1,1.2)$.

We notice that the $\gamma$ decay also leads to power-law relations between the half-life and the released energy [1], with even exponents from $t \propto 1 / Q^{3}, t \propto 1 / Q^{5}$, etc.; this means powerlaw distributions with $\tau \simeq 4 / 3,6 / 5 \ldots$, very close to 1 in all cases, in agreement with the results found for isomeric transitions.

Power-law behaviors as the ones found here for radioactive decays are widely spread in nature. The origin of the abundance of these scaling laws is a topic of great interest in our understanding of complex systems, i.e., systems containing a large number of highly interacting components. Nuclei are a good example of a complex system, as nucleons strongly interact among each other in such a way that no theory is able to predict from first principles which nuclides will be stable. Though one might be tempted to believe that just a general mechanism may be responsible for the ubiquity of power laws in nature, several different explanations have been reported, such as aggregation processes [22,23], intermittency [24], self-organized criticality [3], and multiplicative processes [25,26]; see Refs. [6,18,27] for reviews. As discussed above, the power laws of nuclear half-lives are simply related to the 
extremely steep relationships between half-life and energy, which translate small changes in energy to variations of orders of magnitude in half-life.

\section{OTHER POWER LAWS AND OTHER FITS}

Going back to data analysis, one can perform the same study with the half-lives of elementary particles. We have compiled a list of 131 baryons and mesons, with $t$ ranging from $10^{-25} \mathrm{~s}$ to about $10 \mathrm{~min}$ (for the neutron). The direct application of the Clauset et al.'s method, described above, leads to a pure powerlaw distribution with $a=2.4 \times 10^{-14} \mathrm{~s}$ and $\tau=1.13 \pm 0.03$, comprising 19 particles and with $p=7 \% \pm 2.5 \%$. Although this is in the limit of rejection, it is striking that the exponent $\tau$ is essentially the same as for the radionuclides. The similarity in some disintegration processes could explain this behavior.

Interestingly, other power-law ranges exist for the distribution of nuclear half-lives. For the whole data set, imposing $b<1$ s (which corresponds to the region of short halflives), we find the optimum values $a=5.0 \times 10^{-5} \mathrm{~s}$ and $b=0.32 \mathrm{~s}$, with $\tau=0.65 \pm 0.02, d_{m}=0.023$, and $p=$ $40 \% \pm 5 \%$, comprising 460 radionuclides. We can conclude that a second (or a third), flatter power-law regime is present in the data, although incompleteness of the data for short half-lives can affect the range of this regime; see Table I.

The transition between the short and long half-life powerlaw regimes shows a convex shape in log-log scale that is well modeled by a (truncated) lognormal distribution from about $10^{-2}$ to $10^{5} \mathrm{~s}$, with the parameters of the underlying (untruncated) normal distribution $(x \equiv \ln t)$ given by $\mu \simeq 3.4$ and $\sigma^{2} \simeq 30$, if $t$ is measured in seconds. Again, there is an overlap region between the different distributions, in which a power-law fit and the log-normal fit are both valid.

We are not interested in determining precisely when one distribution transforms into another, but, more importantly, we have tested that the lower truncated lognormal distribution is not preferred in front of the power law for values of $t$ above $100 \mathrm{~s}$. This has been done by the straightforward adaptation of the uniformly most powerful unbiased test proposed in Ref. [28]. The main idea is that a power-law distribution constitutes a special case of a (truncated) lognormal distribution, achieved when $\sigma \rightarrow \infty$ and $\mu \rightarrow-\infty$ but with $\sigma \ll|\mu|$ in such a way that the power-law exponent turns out to be $\tau=1+|\mu| / \sigma^{2}$. A likelihood ratio test evaluates if other parameters for the lognormal are preferred. In our case, the power-law fit cannot be rejected. The nonpreference of the lognormal can be extended to other related distributions [28].

\section{CONCLUSION}

In summary, the distribution of half-lives of the radionuclides has a power-law tail, with an exponent slightly greater than 1 , due to the abrupt relationship between decay rate and released energy for the different transitions analyzed. The broad range of scales covered by the distribution and the scarce number of nuclides in the right-most part of it makes difficult the use of standard statistical tools, and even a recently introduced fitting procedure [7] fails to detect the power law. Careful analysis is necessary when dealing with power-law distributions with such small exponents.

\section{ACKNOWLEDGMENTS}

The authors acknowledge valuable information flow from A. Clauset, A. Deluca, R. D. Malmgren, P. Puig, and K. Sneppen and appreciate the efforts of the late P. Bak, B. B. Mandelbrot, and M. Schroeder to point out the relevance of power-law statistics. F. F. was supported by a grant from the CRM. Spanish projects involved: FIS2009-09508, FIS200913370-C02-01, and 2009SGR-164. A.C. also participates in the Consolider i-Math project.

\section{APPENDIX}

Our fitting procedure and the testing of the goodness of fit follow closely the ideas reported by Clauset et al. [7] and are explained in more detail in the supplementary information of Ref. [14]. Here we just provide an overview of the basic method; further modifications are specified in the main text.

The maximum-likelihood (ML) estimation of the exponent of a power-law distribution of the form $D(t)=(\tau-$ 1) $a^{\tau-1} / t^{\tau}$, with $t>a$ (and $a$ known) is given by

$$
\tau=1+\frac{1}{\ln \frac{G_{a}}{a}}
$$

where $G_{a}$ is the geometric mean of the data above the cutoff, $\ln G_{a} \equiv N_{a}^{-1} \sum_{t \geqslant a} \ln t$, and $N_{a}$ is the number of such data. It can be easily shown that for true power-law distributed data the ML estimator of the exponent (minus 1) follows an inverse $\gamma$ distribution, with mean $N_{a}(\tau-1) /\left(N_{a}-1\right)$ asymptotically equal to the true exponent (minus 1 ) and a standard deviation given by

$$
\varepsilon=\frac{\tau-1}{\left(1-N_{a}^{-1}\right) \sqrt{N_{a}-2}} \rightarrow \frac{\tau-1}{\sqrt{N_{a}}} ;
$$

note that the uncertainty of the exponent goes to zero as its value goes to 1 (which, on the other hand, is forbidden by normalization).

As the estimated value of $\tau$ depends only on the geometric mean of the data, any data set with the same $G_{a}$ will lead to the same value of the exponent and the same uncertainty (if $N_{a}$ is fixed), independently of whether the data come from a power law or from an alternative distribution. Providing a goodness of the fit is then necessary. For that purpose, one can use the KS distance [11], defined as,

$$
d=\max _{\forall t \geqslant a}\left|S(t)-S_{\mathrm{emp}}(t)\right|,
$$

i.e., as the maximum absolute difference between the empirical cumulative distribution function, $S_{\mathrm{emp}}(t)$, and the fitted cumulative distribution function, $S(t)$. For simplicity, we identify the cumulative distribution with $S(t)=\int_{t}^{\infty} D(t) d t$ (that is, we use the complementary cumulative distribution, which does not affect the definition of the KS distance), and 

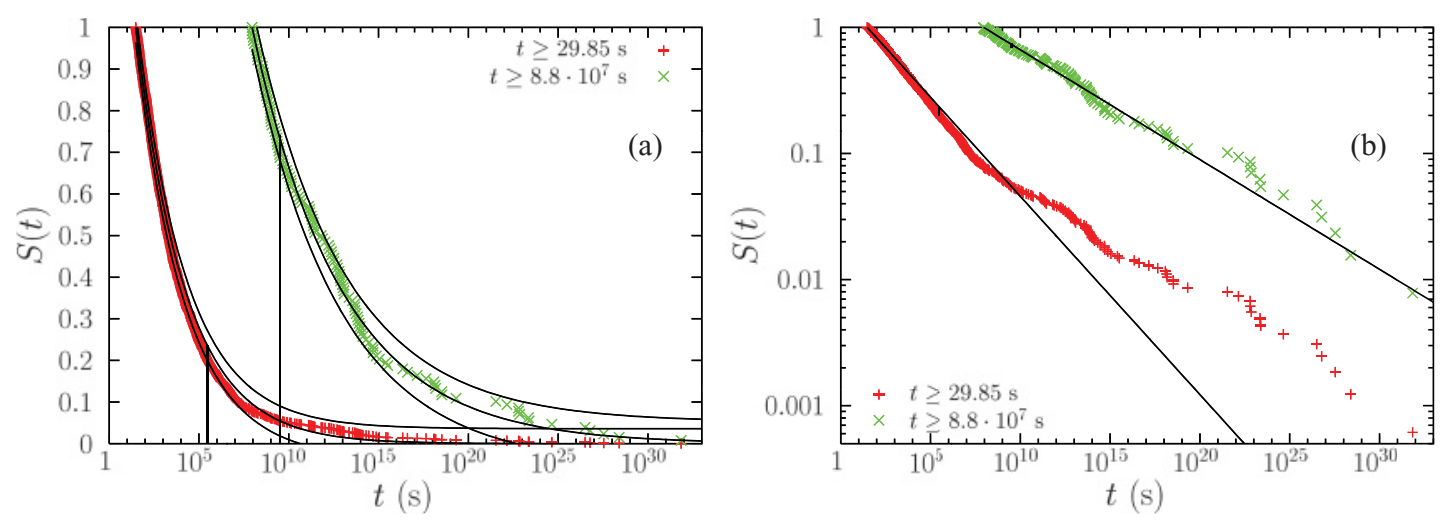

FIG. 3. (Color online) (a) Procedure for calculating the KS distance, exemplified with two data sets. In each case, both the empirical and the fitted cumulative distribution functions are shown. The KS distance $d_{m}$ is the maximum vertical difference between both curves, and the value of the half-life corresponding to this maximum is shown as a vertical line. Two additional curves, given by $S(t) \pm d_{m}$, are also shown for clarity sake. The first data set, on the left, is for the half-lives with $t \geqslant a=29.85 \mathrm{~s}$, corresponding to the Clauset $e t$ al.'s solution, which yields a bad fit, with $p=0$. The second data set, on the right, is for $t \geqslant 8.8 \times 10^{7} \mathrm{~s}$, and although it yields a larger KS distance (0.052 versus 0.036 ), leads to a much better power-law fit, as it is more clearly seen on (b) on a doubly logarithmic scale.

then

$$
S(t)=\left(\frac{a}{t}\right)^{\tau-1},
$$

while $S_{\mathrm{emp}}(t)=n(t) / N_{a}$, with $n(t)$ the number of data at or above $t$ (and not below $a$ ). In this way, large values of $d$ denote bad fits, whereas small values correspond to good fits, the boundary between large and small will be made more precise below. Figure 3 illustrates the computation of the KS distance for the nuclear half-lives with two special values of $a$, taken from Table I.

The key of the Clauset et al.'s recipe is to consider the cutoff $a$ not as a fixed quantity but as a parameter that needs to be estimated from data as well. The previous procedure (fitting of $\tau$ and calculation of $\mathrm{KS}$ distance) is then repeated for all possible $a$ values, and the selected one corresponds to the one that minimizes the KS distance, i.e., $d_{m}=\min _{\forall a} d$. This leads automatically to one value of $a$ and $\tau$.

In order to quantify the goodness of fit we need to compare $d_{m}$ with the results for true power-law distributed data. We simulate synthetic data sets, power law distributed for $t \geqslant a$, using

$$
t=\frac{a}{(1-u)^{1 /(\tau-1)}},
$$

with probability $N_{a} / N$ and $u$ a uniform random number between 0 and 1 , and bootstraping the empirical data set for $t<a$, with probability $N_{a} / N$ (and $N$ is the total number of data, $\forall t$ ). Then, we apply exactly the same ML estimation of the exponent and calculation of the KS distance to each synthetic data set. We stress that the KS distance is computed between the simulated distribution and its ML fit (not the fit of the empirical distribution, which provides the parameters for the simulation). In this way we end with a distribution of values of $d_{m}$, which allows one to compute the $p$ value of the fit, as the ratio between the number of simulations with $d_{m}$ above the empirical one and the total number of simulations.

If we generalize the method to an upper truncated powerlaw distribution,

$$
D(t)=\frac{\tau-1}{1-r^{\tau-1}} \frac{a^{\tau-1}}{t^{\tau}}
$$

defined in $[a, b)$, with $r=a / b$, then the previous formulas need to be replaced by

$$
\begin{aligned}
& \frac{1}{\tau-1}+\frac{r^{\tau-1} \ln r}{1-r^{\tau-1}}-\ln \frac{G_{a b}}{a}=0, \\
& \sqrt{N_{a b}} \varepsilon=\left[\frac{1}{(\tau-1)^{2}}-\frac{r^{\tau-1} \ln ^{2} r}{\left(1-r^{\tau-1}\right)^{2}}\right]^{-1 / 2}, \\
& S(t)=\frac{1}{1-r^{\tau-1}}\left[\left(\frac{a}{t}\right)^{\tau-1}-r^{\tau-1}\right], \\
& t=\frac{a}{\left[1-\left(1-r^{\tau-1}\right) u\right]^{1 /(\tau-1)}},
\end{aligned}
$$

for the ML estimation of $\tau$, its asymptotic standard deviation (taken from Ref. [29]), the complementary cumulative distribution, and the simulated values of the variable in the power-law region, $a \leqslant t<b$ (taken with probability $\left.N_{a b} / N\right)$, respectively.
[1] K. S. Krane, Introductory Nuclear Physics (Wiley, New York, 1988).

[2] M. Schroeder, Fractals, Chaos, Power Laws (Freeman, New York, 1991).
[3] P. Bak, How Nature Works: The Science of SelfOrganized Criticality (Copernicus, New York, c15 1996).

[4] B. D. Malamud, Phys. World 17, 31 (2004). 
[5] K. Christensen and N. R. Moloney, Complexity and Criticality (Imperial College Press, London, 2005).

[6] D. Sornette, Critical Phenomena in Natural Sciences, 2nd ed. (Springer, Berlin, 2004).

[7] A. Clauset, C. R. Shalizi, and M. E. J. Newman, SIAM Rev. 51, 661 (2009).

[8] S. Y. F. Chu, L. P. Ekström, and R. B. Firestone, Lund/LBNL Nuclear Data Search web page [http://nucleardata. nuclear.lu.se/nucleardata/toi], version 2.0, February 1999.

[9] P. de Marcillac, N. Coron, G. Dambier, J. Leblanc, and J.-P. Moalic, Nature 422, 876 (2003).

[10] S. Hergarten, Self-Organized Criticality in Earth Systems (Springer, Berlin, 2002).

[11] W. H. Press, S. A. Teukolsky, W. T. Vetterling, and B. P. Flannery, Numerical Recipes in FORTRAN, 2nd ed. (Cambridge University Press, Cambridge, UK, 1992).

[12] R. D. Malmgren, D. B. Stouffer, A. E. Motter, and L. A. N. Amaral, Proc. Natl. Acad. Sci. USA 105, 18153 (2008).

[13] S. M. Burroughs and S. F. Tebbens, Pure Appl. Geophys. 158, 741 (2001).

[14] A. Corral, A. Ossó, and J. E. Llebot, Nat. Phys. 6, 693 (2010).

[15] O. Peters, A. Deluca, A. Corral, J. D. Neelin, and C. E. Holloway, J. Stat. Mech. (2010) P11030.
[16] J. Miller, Phys. Today 63(6), 11 (2010).

[17] W. S. C. Williams, Nuclear and Particle Physics (Oxford Science, New York, 1997).

[18] M. E. J. Newman, Contemp. Phys. 46, 323 (2005).

[19] D. H. Perkins, Introduction to High Energy Physics, 4th ed. (Cambridge University Press, Cambridge, 2000).

[20] S. Coles, An Introduction to Statistical Modeling of Extreme Values (Springer, London, 2001).

[21] P. Embrechts, C. Klüppelberg, and T. Mikosch, Modelling Extremal Events (Springer, Berlin, 1997).

[22] T. Vicsek, P. Meakin, and F. Family, Phys. Rev. A 32, 1122 (1985).

[23] J. Camacho and R. V. Solé, Europhys. Lett. 55, 554 (2001).

[24] Y. B. Zeldovich, Zh. Eksp. Teor. Fiz. 32, 552 (1957).

[25] W. J. Reed and B. D. Hughes, Phys. Rev. E 66, 067103 (2002).

[26] A. Saichev, Y. Malevergne, and D. Sornette, Theory of Zipfs Law and of General Power Law Distributions with Gibrats law of Proportional Growth. Lecture Notes in Economics and Mathematical Systems (Springer-Verlag, Berlin, 2009).

[27] M. Mitzenmacher, Internet Math. 1(2), 226 (2004).

[28] J. del Castillo and P. Puig, J. Am. Stat. Assoc. 94, 529 (1999).

[29] I. B. Aban, M. M. Meerschaert, and A. K. Panorska, J. Am. Stat. Assoc. 101, 270 (2006). 